\title{
Geodesic flows in a charged black hole spacetime with quintessence
}

\author{
Hemwati Nandan ${ }^{1, \mathrm{a}}$, Rashmi Uniyal ${ }^{1,2, \mathrm{~b}}$ \\ ${ }^{1}$ Department of Physics, Gurukul Kangri Vishwavidyalaya, Haridwar, Uttarakhand 249 404, India \\ 2 Department of Physics, Government Degree College, Narendranagar, Tehri Garhwal, Uttarakhand 249 175, India
}

Received: 23 December 2016/ Accepted: 29 July 2017 / Published online: 17 August 2017

(C) The Author(s) 2017. This article is an open access publication

\begin{abstract}
We investigate the evolution of timelike geodesic congruences, in the background of a charged black hole spacetime surrounded by quintessence. The Raychaudhuri equations for three kinematical quantities namely the expansion scalar, shear and rotation along the geodesic flows in such spacetime are obtained and solved numerically. We have also analysed both the weak and the strong energy conditions for the focussing of timelike geodesic congruences. The effect of the normalisation constant $(\alpha)$ and the equation of state parameter $(\varepsilon)$ on the evolution of the expansion scalar is discussed, for the congruences with and without an initial shear and rotation. It is observed that there always exists a critical value of the initial expansion below which we have focussing with smaller values of the normalisation constant and the equation of state parameter. As the corresponding values of both of these parameters are increased, no geodesic focussing is observed. The results obtained are then compared with those of the Reissner Nordström and Schwarzschild black hole spacetimes as well as their de Sitter black hole analogues accordingly.
\end{abstract}

\section{Introduction}

The Black Holes (BHs) are the most fascinating objects in the universe those arise in Einstein's General Relativity (GR), a classical theory of gravity proposed by Einstein about a century ago [1-6]. The Schwarzschild metric obtained in GR was the first unique solution to Einstein's field equations in vacuum with a spherically symmetric matter distribution, which represents the simplest spacetime of a black hole $(\mathrm{BH})$ having mass but no charge and spin $[7,8]$. There are, however, other BH spacetimes emerging as solutions of Einstein's field equations in GR having charge/or spin with mass such as the Reissner-Nordström BH $[9,10]$, the Kerr BH [11], and

\footnotetext{
a e-mail: hnandan@iucaa.ernet.in

b e-mail: rashmiuniyal001@gmail.com
}

the Kerr-Newmann BH spacetimes [12] along with the BH spacetimes in alternative theories of gravity like string theory.

In GR, the curvature plays crucial role to understand the geometric effect of curved spacetime. The study of geodesics alongwith their deformations in the background of a given spacetime is an elegant way to describe the underlying geometry of that particular spacetime $[1-3,5,6,13,30]$. A number of studies related to the geodesic motion in the background of various $\mathrm{BH}$ spacetimes have been performed time and again mainly in view of their astrophysical importance [14-29].

The observations from supernovae (Type Ia), cosmic microwave background radiation (CMBR), Baryon acoustic oscillations (BAO) and the Hubble measurements indicates that our universe appears to be expanding at an increasing rate. The driving force behind such an accelerating universe is believed to be some unknown form of energy with a large negative pressure which is known as dark energy. There are several candidates for dark energy, such as cosmological constant [30,31], phantom [32-35], quintessence $[36,37]$, K-essence $[38,39]$ and quintom [40-42] with various models subjected to the different values of the equation of state (EOS) parameter $(\varepsilon)$ which relates the energy density to the pressure. The quintessence scalar field model as an alternative to dark energy is one of the most popular models with the EOS parameter lying in the range $-1<\varepsilon<-1 / 3$.

It would therefore be quite interesting to study the geodesics and their deformations in the background of a charged BH spacetime surrounded by quintessence to see the effect of dark energy, if any, on the universe locally. It is also important to look on the matter distribution which causes this spacetime such that the Einstein equations hold and to identify the interesting regions in the spacetime in view of the weak energy condition (WEC) and strong energy condition (SEC).

In the present paper, we study the geodesic flows and deformations alongwith energy conditions around a charged BH spacetime surrounded by quintessence by solving the evolution (i.e. Raychaudhuri) equations as an initial value 
problem for expansion scalar, shear and rotation (ESR variables) numerically. First, we briefly review the spacetime used in the next section. In Sect. 3, the nature of effective potential is discussed. Section 4 deals with the discussion of the kinematics of geodesic flows and visualisation of ESR. Finally, the results are summarized in Sect. 5.

\section{The charged BH spacetime surrounded by quintessence}

We consider a charged $\mathrm{BH}$ surrounded by quintessence with the EOS parameter $\varepsilon=\frac{p_{\Phi}}{\rho_{\Phi}}$. For the static spherically symmetric quintessence surrounding a $\mathrm{BH}$, the energy density of quintessence scalar field $(\Phi)$ reduces to the following form [43]:

$\rho_{\Phi}=-\frac{\alpha}{2} \frac{3 \varepsilon}{r^{3(1+\varepsilon)}}$,

where the allowed values for $\varepsilon$ lies between $-1<\varepsilon<-\frac{1}{3}$ [43] and $\alpha$ is the normalisation constant. The energy density of scalar field, $\rho_{\Phi}$ is always a positive quantity so $\varepsilon$ has a negative value while the normalisation factor $\alpha$ should be a positive quantity. Based on such standpoints, the metric of a charged $\mathrm{BH}$ then reads

$\mathrm{d} s^{2}=f(r) \mathrm{d} t^{2}-\frac{1}{f(r)} \mathrm{d} r^{2}-r^{2}\left(\mathrm{~d} \theta^{2}+\sin ^{2} \theta \mathrm{d} \phi^{2}\right)$,

where

$f(r)=1-\frac{2 M}{r}+\frac{Q^{2}}{r^{2}}-\frac{\alpha}{r^{3 \varepsilon+1}}$,

here $M$ and $Q$ represent the mass and charge of the $\mathrm{BH}$ respectively. The above metric (2) represents a $\mathrm{BH}$ for $M>$ $Q$, an extremal BH for $M=Q$ and a naked singularity for $M<Q$ (for a complete discussion of the horizon structure for this spacetime see [44]). The metric reduces to a Reissner-Nordström black hole (RNBH) in the limit $\alpha=0$, which further reduces to Schwarzschild black hole (SBH) in the absence of charge. In addition to this, with $\varepsilon=-1$, it also reproduces the corresponding BH spacetimes with cosmological constant. The geodesic equations for the metric (2) are given by

$$
\begin{aligned}
& \ddot{t}+\frac{f^{\prime}(r)}{f(r)} \dot{r} \dot{t}=0, \\
& \ddot{r}+\left[\frac{f^{\prime}(r) \dot{t}^{2}+f^{-1}(r)^{\prime} \dot{r}^{2}-2 r \dot{\theta}^{2}-2 r \sin ^{2} \theta \dot{\phi}^{2}}{2 f^{-1}(r)}\right]=0,
\end{aligned}
$$

$\ddot{\theta}+\frac{2}{r} \dot{r} \dot{\theta}-\cos \theta \sin \theta \dot{\phi}^{2}=0$,

$\ddot{\phi}+\frac{2}{r} \dot{r} \dot{\phi}+2 \cot \theta \dot{\theta} \dot{\phi}=0$,

where the prime denotes the differentiation with respect to $r$. The first integral of the geodesic Eqs. (3) and (6) on equatorial plane (i.e. $\theta=\pi / 2$ ) read

$\dot{t}=\frac{E}{1-\frac{2 M}{r}+\frac{Q^{2}}{r^{2}}-\frac{\alpha}{r^{3 \varepsilon+1}}}$,

$\dot{\phi}=\frac{L}{r^{2}}$,

where $E$ and $L$ are the integrating constants which correspond to the conserved total energy and angular momentum per unit mass respectively for a test particle. Using the constraint $u^{\mu} u_{\mu}=-1$, the expression for radial velocity $\left(u^{r}=\right.$ $\dot{r}$ ) can now be obtained:

$\dot{r}^{2}=E^{2}-V_{\mathrm{eff}}$,

where $V_{\text {eff }}$ is defined as an effective potential and is expressed as

$V_{\mathrm{eff}}=\left(1-\frac{2 M}{r}+\frac{Q^{2}}{r^{2}}-\frac{\alpha}{r^{3 \varepsilon+1}}\right)\left(\frac{L^{2}}{r^{2}}+1\right)$.

\section{Nature of effective potential}

Figures 1 and 2 represent the nature of effective potential for different values of the parameters involved in. In particular, Fig. 1a-c represent the corresponding nature of effective potential for a $\mathrm{BH}$, extremal $\mathrm{BH}$ and naked singularity respectively. It is evident from the Fig. 2 that the nature of effective potential is qualitatively similar for SBH and that of charged BH with $\varepsilon=-1 / 3$. Meanwhile, for $\varepsilon=-2 / 3$, there exist no minima in the radial plot of effective potential. Hence there are no stable circular orbits for charged $\mathrm{BH}$ surrounded by quintessence. For the circular motion of a test particle, its radial velocity vanishes. Hence using Eqs. (9) and (10) the angular momentum and energy per unit mass for the incoming test particle in circular motion is

$$
\begin{aligned}
P L_{c}^{2}=- & r_{c}^{2}\left(2 M r_{c}^{3 \varepsilon+2}-2 Q^{2} r_{c}^{3 \varepsilon+1}+3 \alpha \varepsilon r_{c}^{2}+\alpha r_{c}^{2}\right), \\
P r_{c}^{2} E_{c}^{2}= & 4 Q^{2} \alpha r_{c}^{2}-8 M \alpha r_{c}^{3}+4 \alpha r_{c}^{4}-2 r_{c}^{3 \varepsilon+5} \\
& -2 Q^{4} r_{c}^{3 \varepsilon+1}-4 Q^{2} r_{c}^{3 \varepsilon+3}-2 \alpha^{2} r_{c}^{-3 \varepsilon+3} \\
& +8 M r_{c}^{3 \varepsilon+4}-8 M^{2} r_{c}^{3 \varepsilon+3}+8 Q^{2} M r_{c}^{3 \varepsilon+2}
\end{aligned}
$$

where $P=3 \alpha(\varepsilon+1) r_{c}^{2}-4 Q^{2} r_{c}^{3 \varepsilon+1}+6 M r_{c}^{3 \varepsilon+2}-2 r_{c}^{3 \varepsilon+3}$ and $L_{c}, E_{c}$ represent the specific angular momentum and energy 


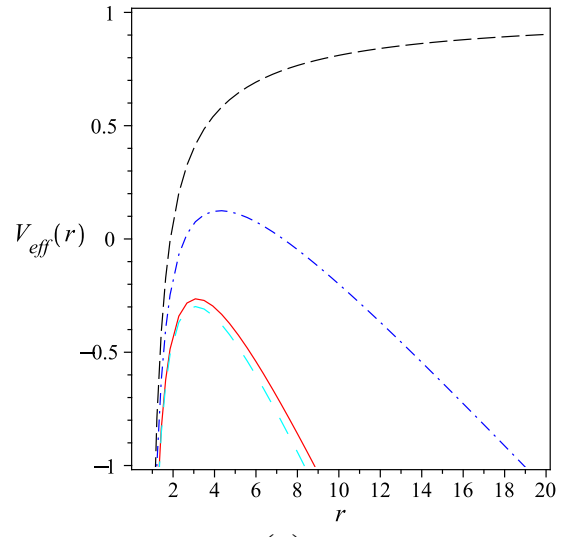

(a) $\mathrm{Q}=0.5$

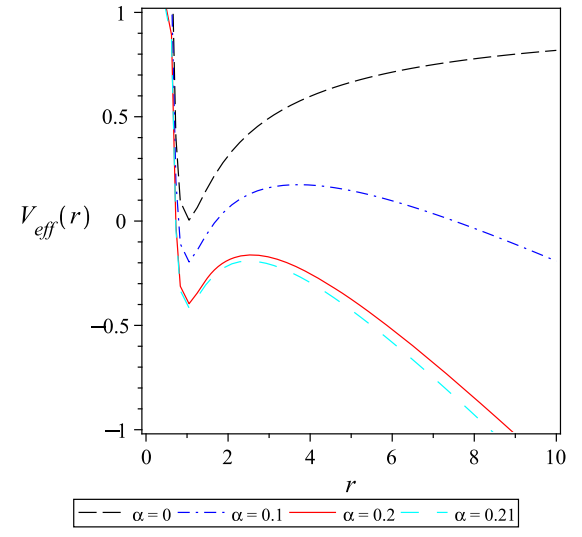

(b) $\mathrm{Q}=1$

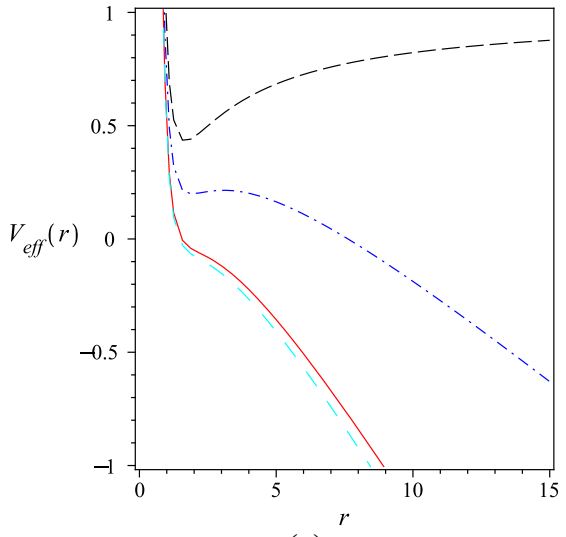

(c) $\mathrm{Q}=1.2$

Fig. 1 The effective potential for $M=L=1$ and $\varepsilon=-2 / 3$

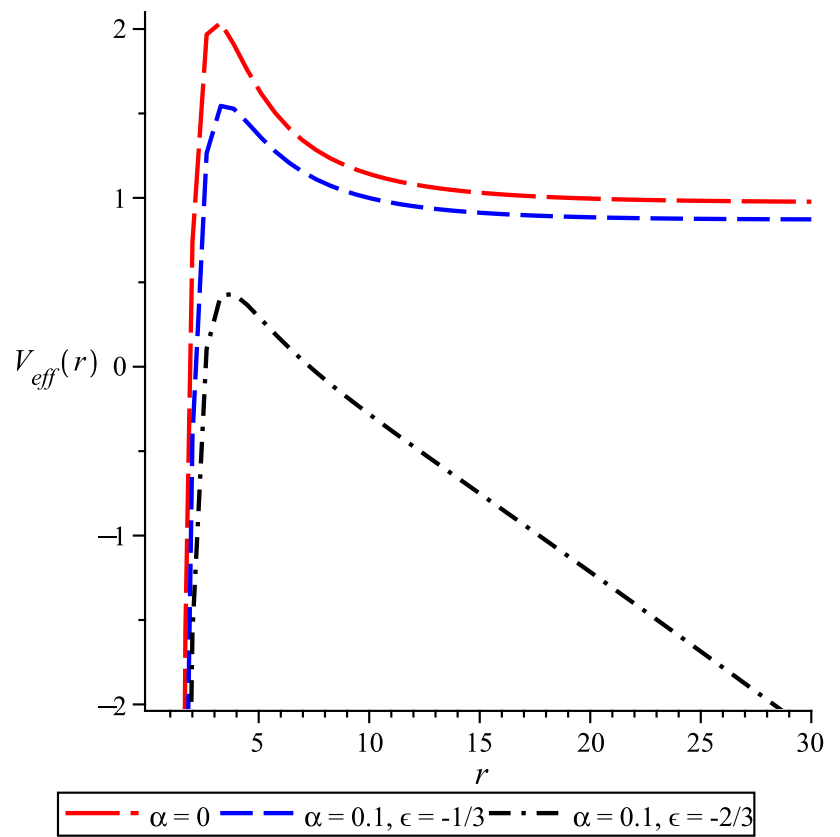

Fig. 2 The effective potential with $M=1, Q=0.5, L=6.5$

per unit mass for massive test particle in circular motion with corresponding radius of circular orbit $r_{c}$.

\section{Geodesic flows}

\subsection{The energy conditions}

The Ricci scalar for the BH spacetime given by Eq. (2) is calculated to be

$$
R=\frac{3 \alpha \varepsilon(1-3 \varepsilon)}{r^{3 \varepsilon+3}},
$$

one may notice that it diverges at $r \rightarrow 0$ and vanishes at $r \rightarrow \infty$. The stress-energy tensor is proportional to

$$
\begin{array}{crrr}
T^{\mu \nu}=-\frac{A}{B r^{2}} & 0 & 0 & 0 \\
0 & \frac{A B}{r^{6 \varepsilon+4}} & 0 & 0 \\
0 & 0 & C & 0 \\
0 & 0 & 0 & C
\end{array}
$$

where

$$
\begin{aligned}
& A=3 \alpha \varepsilon-Q^{2} r^{3 \varepsilon-1}, \\
& B=r^{3 \varepsilon+1}-2 M r^{3 \varepsilon}+Q^{2} r^{3 \varepsilon-1}-\alpha, \\
& C=-\frac{3 \alpha \varepsilon(1+3 \varepsilon)}{2 r^{3 \varepsilon+5}} .
\end{aligned}
$$

In order to analyse the energy conditions, it is convenient to introduce an orthonormal frame that satisfies

$g_{\mu \nu} e_{\alpha}^{\mu} e_{\beta}^{\nu}=\eta_{\alpha \beta}$

where $\eta_{\alpha \beta}=\operatorname{diag}(1,-1,-1,-1)$ is the Lorentzian metric. We consider a choice of orthonormal basis for given metric as $e_{\alpha}^{\mu}=\operatorname{diag}\left(\frac{1}{\sqrt{g_{00}}}, \frac{1}{\sqrt{-g_{11}}}, \frac{1}{\sqrt{-g_{22}}}, \frac{1}{\sqrt{-g_{33}}}\right)$, where the energy momentum tensor can be written in the following form:

$T^{\mu \nu}=\rho e_{0}^{\mu} e_{0}^{\nu}+p_{1} e_{1}^{\mu} e_{1}^{v}+p_{2} e_{2}^{\mu} e_{2}^{\nu}+p_{3} e_{3}^{\mu} e_{3}^{\nu}$.

The WEC for quintessential fields is

$T_{\mu \nu} u^{\mu} u^{\nu} \geq 0 \approx \rho \geq 0, \rho+p_{i}>0$,

and for the given spacetime (2), we have

$T_{\mu \nu} u^{\mu} u^{\nu}=\frac{1}{r^{3 \varepsilon+3}}\left(Q^{2} r^{3 \varepsilon-1}-3 \alpha \varepsilon\right)$ 


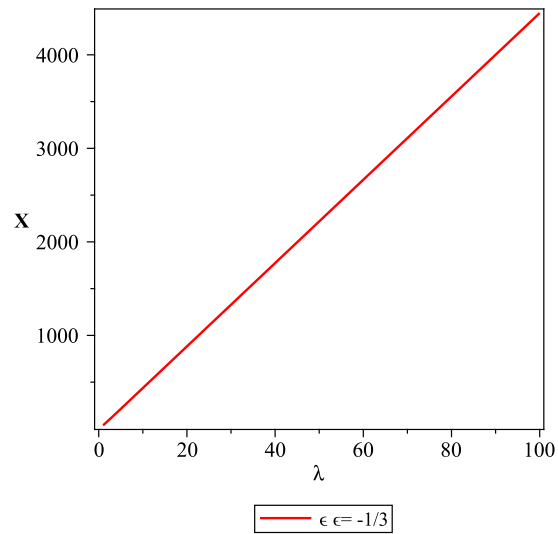

(a)

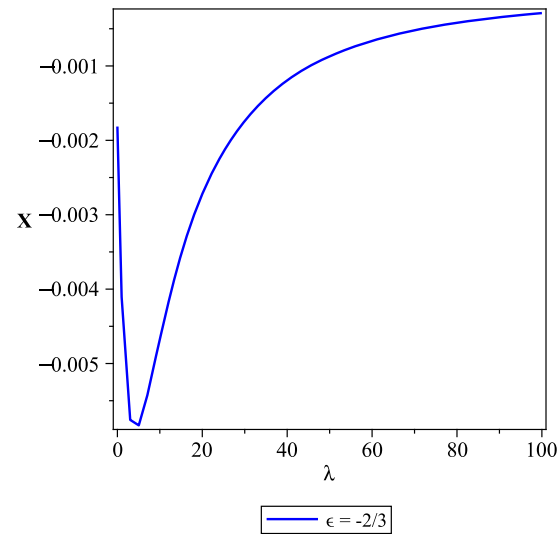

(b)

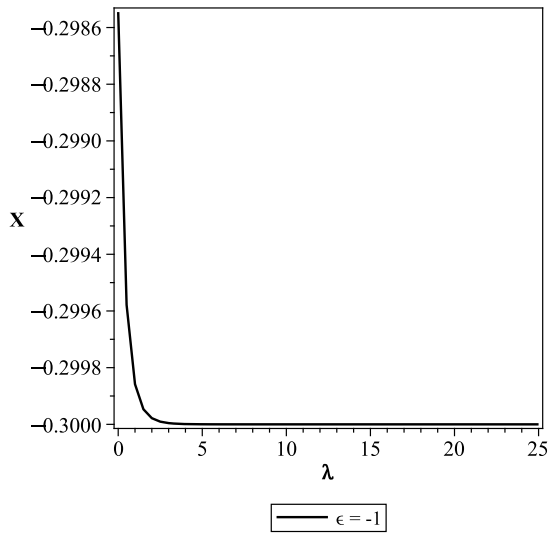

(c)

Fig. 3 Evolution of $X$ with the affine parameter $\lambda$, where $E=0.95, L=6.5, \alpha=0.1, M=1, Q=0.5$ for different values of $\varepsilon$

Hence the WEC simplifies as follows:

$Q^{2} r^{3 \varepsilon-1}-3 \alpha \varepsilon \geq 0$.

However, the SEC reads

$T_{\mu \nu} u^{\mu} u^{\nu} \geq \frac{1}{2} T_{\nu}^{\mu} u^{v} u_{\nu}$

and for the spacetime used it can be written

$\frac{1}{2 r^{3 \varepsilon+1}}\left[2 Q^{2} r^{3 \varepsilon-1}-3 \alpha \varepsilon(3 \varepsilon+1)\right] \geq 0$.

If the WEC represented by Eq. (20) follows, Eq. (22) for the SEC reduces to the following:

$3 \alpha \varepsilon(1-3 \varepsilon) \geq 0$,

hence for $\alpha>0$ and $-\frac{1}{3}<\varepsilon<-1$, the above condition is clearly violated. Both the WEC and the SEC therefore ensure that $r \neq 0$. Hence, locally the attractive nature of gravity may exist there, but on average the quintessential fields have a repulsive nature, which can be visualised in the deformation of geodesic congruences as discussed below.

\subsection{Raychaudhuri equations for ESR variables}

The spacetime given by Eq. (2) can be decomposed into a transverse part i.e. a transverse metric $h_{\mu \nu}$ on a spacelike hypersurface and a longitudinal part $\left(-u_{\mu} u_{\nu}\right)$ as follows:

$h_{\mu \nu}=g_{\mu \nu}+u_{\mu} u_{v}, \quad(\mu, v=0,1,2,3)$.

Here $u^{\mu}$ (a timelike vector field) satisfies the constraint $u^{\mu} u_{\mu}=-1$. One can investigate the evolution of ESR variables on this spacelike hypersurface with $h_{\mu \nu}$ orthogonal to $u^{\mu}$ i.e. $u^{\mu} h_{\mu \nu}=0$, such that it represents the local rest frame of a freely falling observer in given spacetime (2) by using a tensor $B_{\mu \nu}$ which is defined as $B_{\mu \nu}=\nabla_{\nu} u_{\mu}$. For a 4 dimensional spacetime, $B_{\mu \nu}$ can be decomposed as

$B_{\mu \nu}=\frac{1}{3} \theta h_{\mu \nu}+\sigma_{\mu \nu}+\omega_{\mu \nu}$,

here the quantities $\theta, \sigma_{\mu \nu}$ and $\omega_{\mu \nu}$ are known as the expansion scalar, shear tensor and the rotation tensor of the congruence (family) of geodesics defined by $u^{\mu}[3,4]$. These variables can be written explicitly as

$\theta=B_{\mu}^{\mu}$,

$\sigma_{\mu \nu}=\frac{1}{2}\left(B_{\mu \nu}+B_{\nu \mu}\right)-\frac{1}{3} \theta h_{\mu \nu}$,

$\omega_{\mu \nu}=\frac{1}{2}\left(B_{\mu \nu}-B_{\nu \mu}\right)$.

As per their constructional properties, the shear and rotation tensors also satisfy, $h^{\mu v} \sigma_{\mu \nu}=0$ and $h^{\mu \nu} \omega_{\mu \nu}=0$ alongwith $g^{\mu \nu} \sigma_{\mu \nu}=0$ and $g^{\mu \nu} \omega_{\mu \nu}=0$. Since $u^{\mu} \sigma_{\mu \nu}=0$ and $u^{\mu} \omega_{\mu \nu}=0$, both $\sigma_{\mu \nu}$ and $\omega_{\mu \nu}$ are purely spatial in nature (i.e., $\sigma^{\mu \nu} \sigma_{\mu \nu}>0$ and $\omega^{\mu \nu} \omega_{\mu \nu}>0$ ). The evolution equation for the spatial tensor $B_{\mu \nu}$ can also be written as

$\dot{B}_{\mu \nu}+B_{\mu \gamma} B_{v}^{\gamma}=-R_{\mu \eta \nu \delta} u^{\eta} u^{\delta}$,

where $R_{\eta v \mu \delta}$ is the Riemann tensor and the dot (.) represents differentiation w.r.t. the affine parameter $\lambda$. On decomposition of $B_{\mu \nu}$ to the trace, symmetric traceless and antisymmetric parts as in Eqs. (26)-(28), Eq. (29) leads to the Raychaudhuri equations for ESR variables in four dimensions $[3,4]$, 
(a) $M=1, Q=0.5, \varepsilon=-2 / 3, \theta_{0}<\theta_{c}$

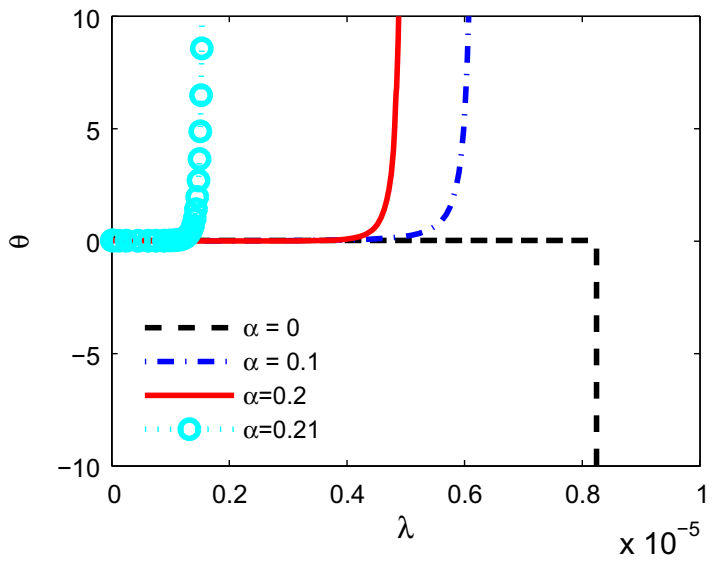

(c) $M=1, Q=1.2, \varepsilon=-2 / 3, \theta_{0}<\theta_{c}$

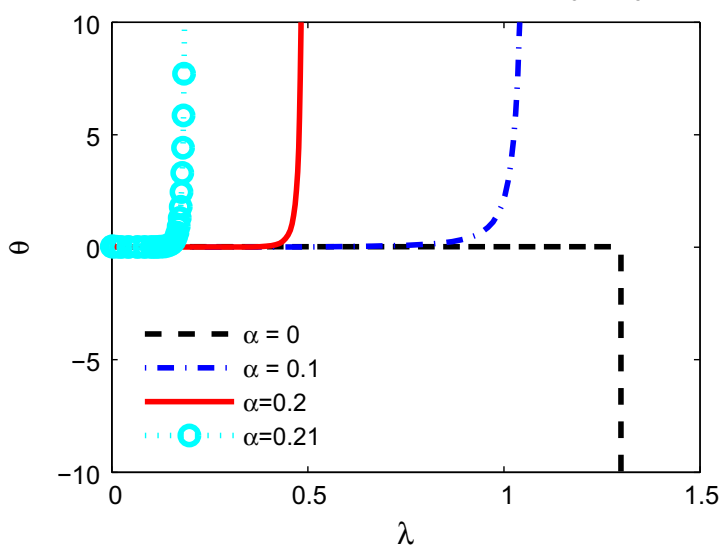

(e) $M=1, Q=1, \varepsilon=-2 / 3, \theta_{0}>\theta_{c}$



(b) $M=1, Q=1, \varepsilon=-2 / 3, \theta_{0}<\theta_{c}$



(d) $M=1, Q=0.5, \varepsilon=-2 / 3, \theta_{0}>\theta_{c}$

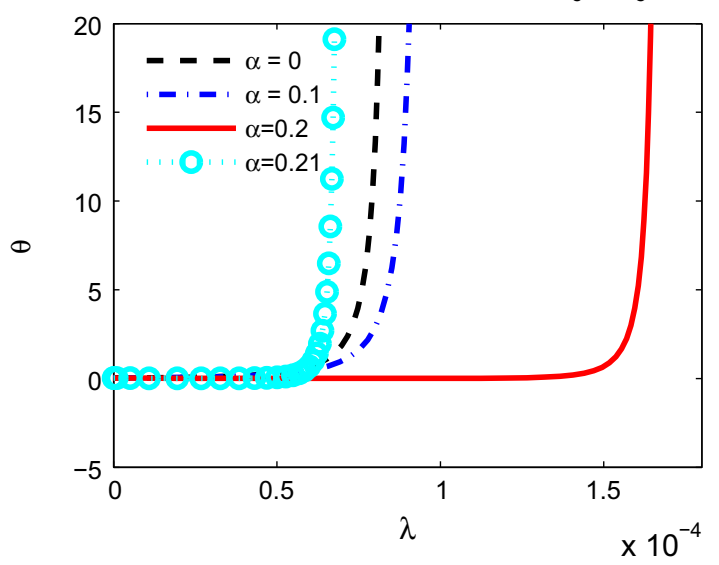

(f) $M=1, Q=1.2, \varepsilon=-2 / 3, \theta_{0}>\theta_{c}$

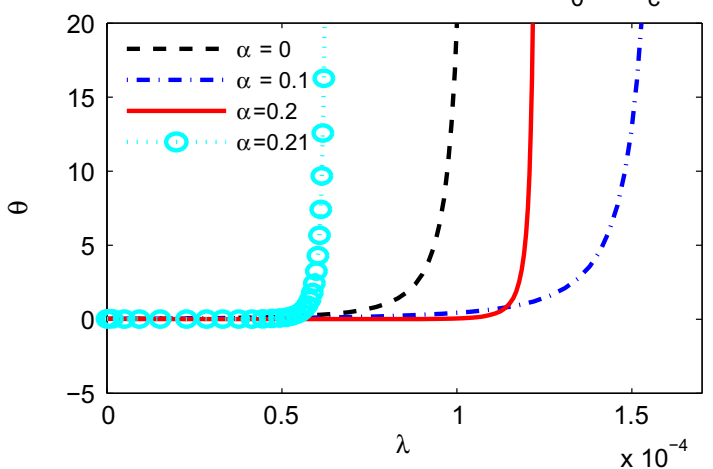

Fig. 4 The evolution of expansion scalar $(\theta)$ with normalisation constant $(\alpha)$ with no initial shear and rotation for different values of charge $(Q)$

$$
\begin{aligned}
& \dot{\theta}+\frac{1}{3} \theta^{2}+\sigma^{2}-\omega^{2}+R_{\mu \nu} u^{\mu} u^{\nu}=0 \\
& \dot{\sigma}_{\mu \nu}+\frac{2}{3} \theta \sigma_{\mu \nu}+\sigma_{\mu \nu} \sigma_{\nu}^{\gamma}+\omega_{\mu \gamma} \omega_{\nu}^{\gamma}+\frac{1}{3}\left(\sigma^{2}-\omega^{2}\right) h_{\mu \nu} \\
& \quad+\mathscr{C}_{\mu \eta \nu \delta} u^{\eta} u^{\delta}-\frac{1}{2} \tilde{\mathscr{R}}_{\mu \nu}=0
\end{aligned}
$$

$\dot{\omega}_{\mu \nu}+\frac{2}{3} \theta \omega_{\mu \nu}+\sigma_{\mu}^{\gamma} \omega_{\gamma \nu}+\omega_{\mu}^{\gamma} \sigma_{\gamma \nu}=0$.

where $\sigma^{2}=\sigma^{\mu \nu} \sigma_{\mu \nu}, \omega^{2}=\omega^{\mu \nu} \omega_{\mu \nu}, \mathscr{C}_{\mu \eta \nu \delta}$ is the Weyl tensor and $\tilde{\mathscr{R}}_{\mu \nu}=\left(h_{\mu \gamma} h_{\nu \delta}-\frac{1}{3} h_{\mu \nu} h_{\gamma \delta}\right) R^{\gamma \delta}$ is the transverse trace-free part of $R_{\mu \nu}$. 


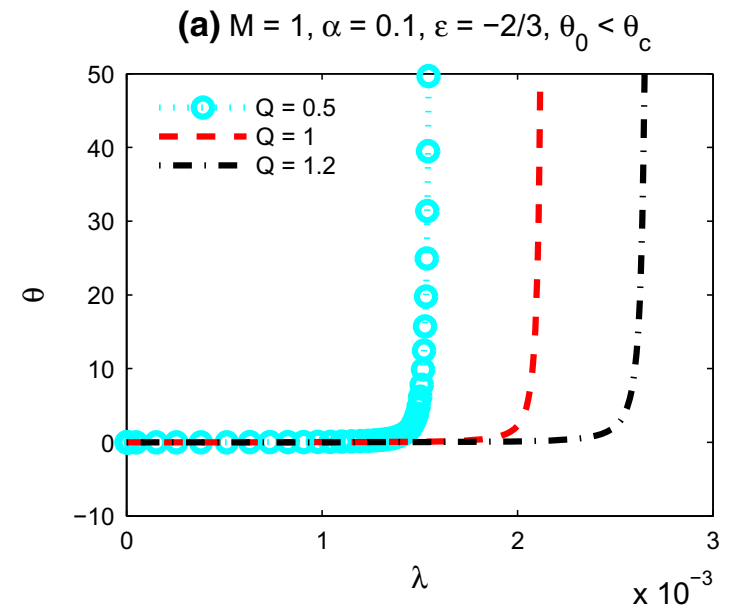

(c) $M=1, Q=0.5, \alpha=0.1, \theta_{0}<\theta_{c}$

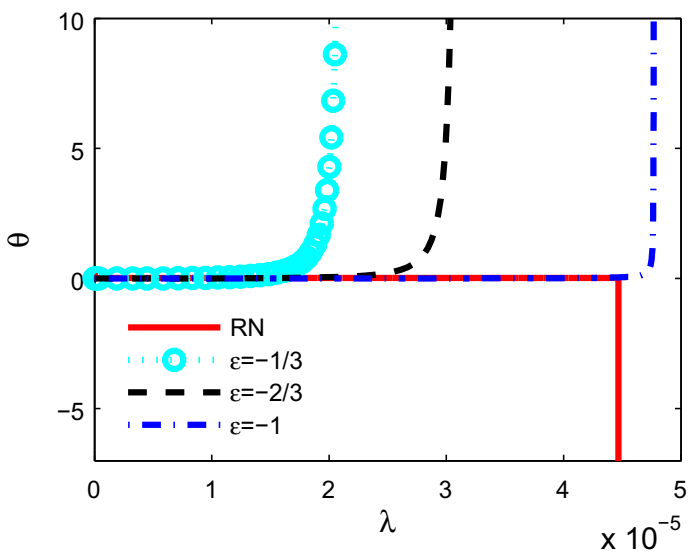

(b) $M=1, \alpha=0.1, \varepsilon=-2 / 3, \theta_{0}>\theta_{c}$

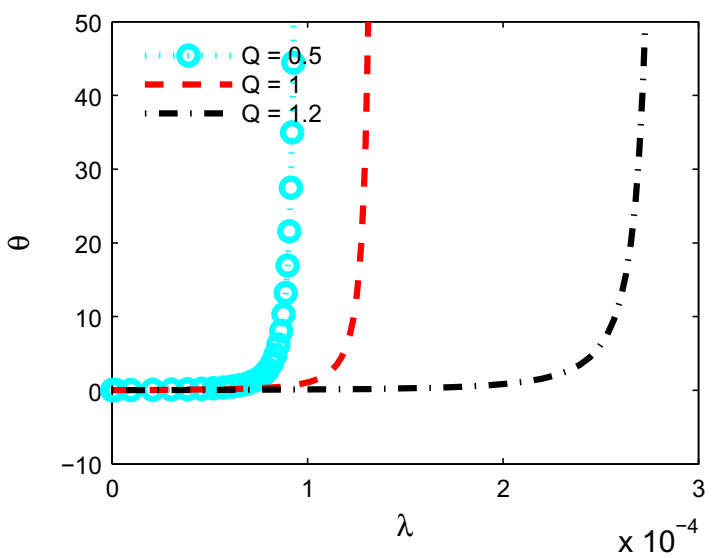

(d) $M=1, Q=0.5, \alpha=0.1, \theta_{0}>\theta_{c}$

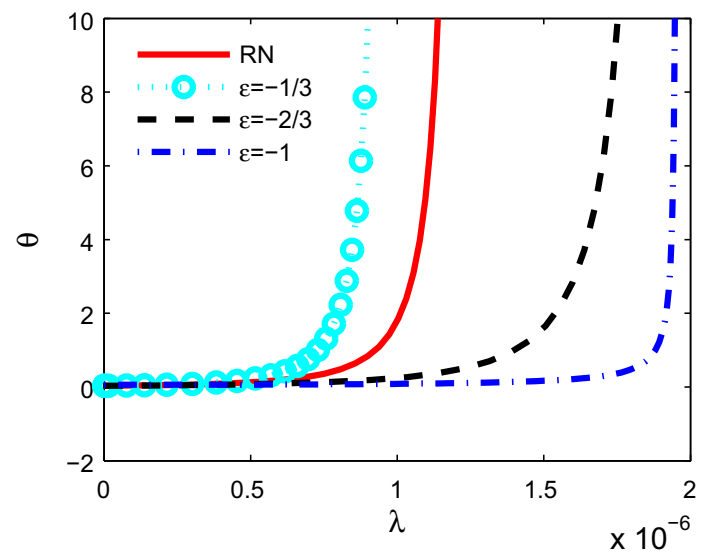

Fig. 5 The evolution of expansion scalar $(\theta)$ with the BH charge $(Q)$ and the EOS parameter $(\varepsilon)$ with no initial shear and rotation

\subsection{Evolution of ESR variables}

In order to represent ESR variables at any point in the geodesic congruence associated with a timelike vector field $u^{\mu}$, let us consider a freely falling (Fermi) normal frame having the basis vectors $E_{\eta}^{\mu}, \eta=0, \ldots, 3$ (with $E_{0}^{\mu}=\hat{u}^{\mu}$ ) which are parallel-transported $[45,46]$. Such frames can be constructed numerically by solving the differential equations $u^{\nu} \nabla_{v} E_{\eta}^{\mu}=0$ (with initial conditions of an orthonormal frame) simultaneously with Eq. (29). The tensor $B_{\mu \nu}$ in the Fermi basis may then be represented as follows:

$$
\begin{aligned}
B_{\mu \nu}= & \left(\frac{1}{3} \theta+\sigma_{11}\right) e_{\mu}^{1} e_{\nu}^{1}+\left(\frac{1}{3} \theta+\sigma_{22}\right) e_{\mu}^{2} e_{v}^{2} \\
& +\left(\frac{1}{3} \theta-\sigma_{11}-\sigma_{22}\right) e_{\mu}^{3} e_{\nu}^{3}+\left(\sigma_{12}-\omega_{3}\right) e_{\mu}^{1} e_{\nu}^{2} \\
& +\left(\sigma_{21}+\omega_{3}\right) e_{\mu}^{2} e_{\nu}^{1}+\left(\sigma_{13}+\omega_{2}\right) e_{\mu}^{1} e_{v}^{3} \\
& +\left(\sigma_{31}-\omega_{2}\right) e_{\mu}^{3} e_{\nu}^{1}+\left(\sigma_{23}-\omega_{1}\right) e_{\mu}^{2} e_{\nu}^{3} \\
& +\left(\sigma_{32}+\omega_{1}\right) e_{\mu}^{3} e_{v}^{2}
\end{aligned}
$$

where $e_{\mu}^{\eta}$ are co-frame basis which satisfy the relation $e_{\mu}^{\eta} E_{\beta}^{\mu}=\delta_{\beta}^{\eta}$. The ESR variables can now be constructed from the evolution tensor (33), using the basis vectors $E_{\eta}^{\mu}$, as described in [45]. In order to understand the focussing and defocussing behaviour of a timelike geodesic congruence, let us further redefine the expansion scalar as $\theta=3 \dot{F} / F$. Equation (32) may now be expressed in the form of the following Hill-type equation:

$\ddot{F}+X F=0$,

where $X=\left(\sigma^{2}-\omega^{2}+R_{\mu \nu} u^{\mu} u^{\nu}\right) / 3$ with $\sigma^{2}=2\left(\sigma_{11}^{2}+\sigma_{22}^{2}+\right.$ $\left.\sigma_{12}^{2}+\sigma_{13}^{2}+\sigma_{23}^{2}+\sigma_{11} \sigma_{22}\right)$ and $\omega^{2}=2\left(\omega_{1}^{2}+\omega_{2}^{2}+\omega_{3}^{2}\right)$. One may note that the Raychaudhuri scalar $R_{\mu \nu} u^{\mu} u^{\nu}$ is zero for the pure SBH case. It is evident from (34) that, for $F \rightarrow 0$ in finite time, we have a finite time singularity in $\theta$ with focussing (defocussing) if $\dot{F}<0(\dot{F}>0)$. The signature of $X$ is thus decisive as we examine focussing/defocussing in view of the critical values for the initial condition on expansion scalar i.e. $\theta_{0}[45,46]$. When $X$ is positive definite (i.e., $\sigma^{2}+$ 
(a) $\varepsilon=-1 / 3, \theta_{0}<\theta_{c}$

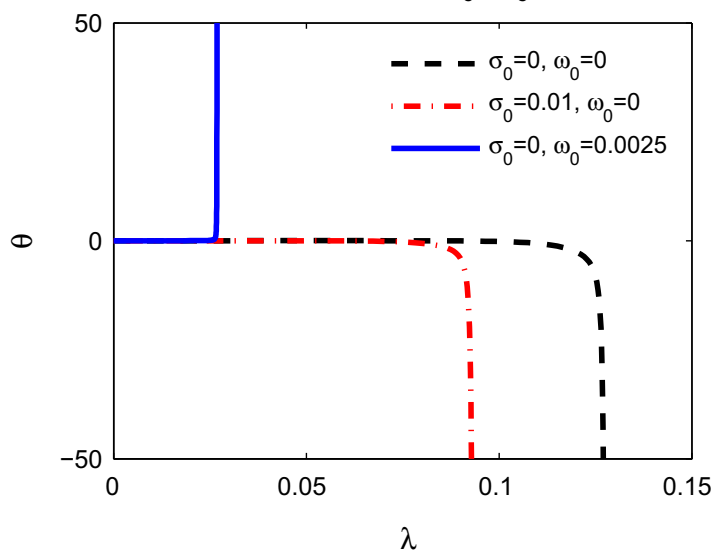

(c) $\varepsilon=-2 / 3, \theta_{0}<\theta_{c}$

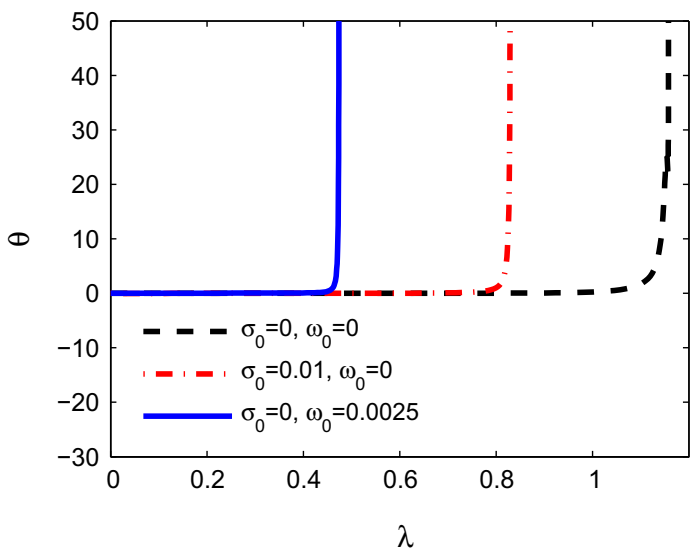

(e) $\varepsilon=-1, \theta_{0}<\theta_{c}$

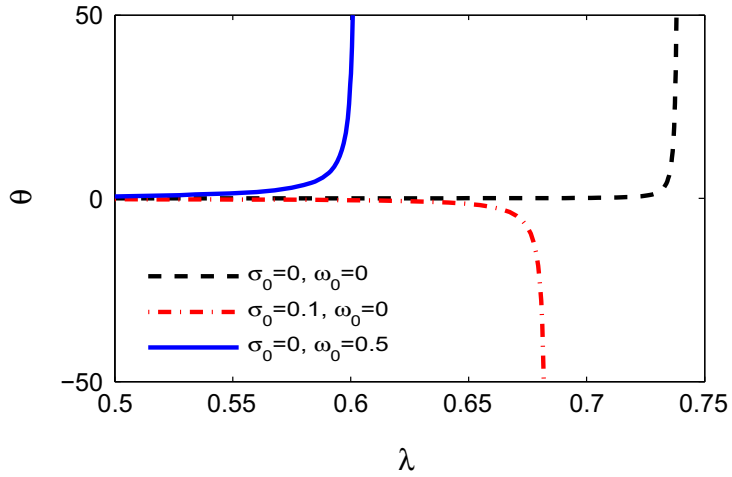

(b) $\varepsilon=-1 / 3, \theta_{0}>\theta_{c}$

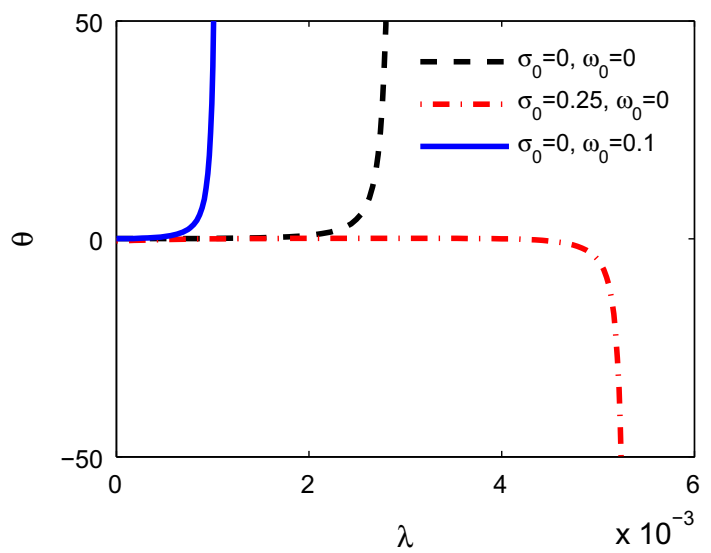

(d) $\varepsilon=-2 / 3, \theta_{0}>\theta_{c}$



(f) $\varepsilon=-1, \theta_{0}>\theta_{\mathrm{c}}$

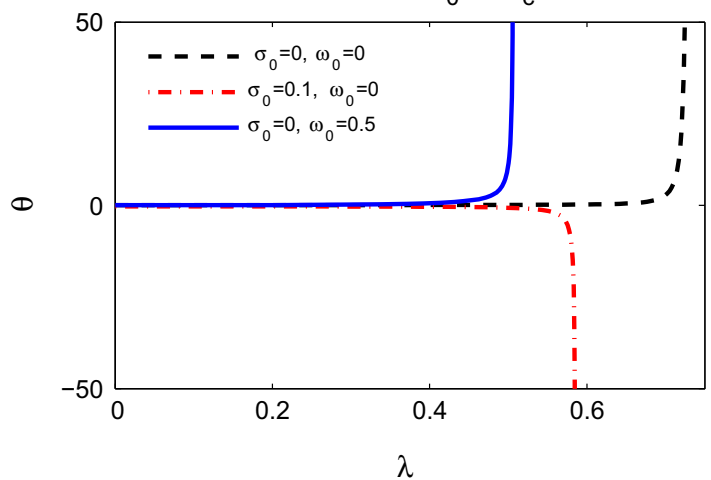

Fig. 6 The evolution of expansion scalar $(\theta)$ with the affine parameter $(\lambda)$ for different values of the EOS parameter $(\varepsilon)$ and initial conditions on ESR variables

$R_{\mu \nu} u^{\mu} u^{\nu}>\omega^{2}$ ), there exist conjugate points and geodesic focussing/defocussing takes place accordingly. On the other hand, no finite time singularity exists for an initially noncontracting congruence (i.e., $\theta_{0} \geq 0$ ) in the case that $X$ is negative definite. For $\theta_{0}<0$, there exists a critical value below which focussing/defocussing will take place. From
Fig. 3, one may easily notice the signature of $X$ for different values of $\varepsilon$, which in turn reflects the non-attractive behaviour of the quintessential fields in case of $\varepsilon=-2 / 3$ and $\varepsilon=$ -1 without initial shear and rotation. However, in view of a positive definite value of $X$ for the case of $\varepsilon=-1 / 3$, geodesic focussing may even occur without initial shear and 
(a) $\theta_{0}<\theta_{c}$

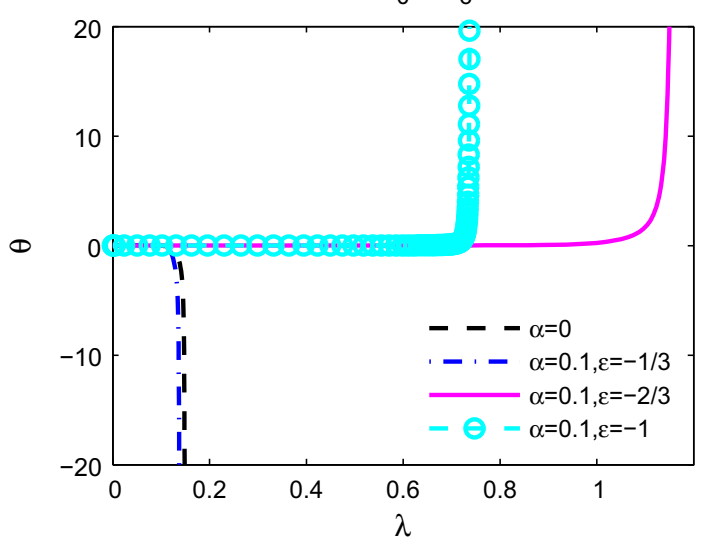

(c) $\varepsilon=-1 / 3, \theta_{0}<\theta_{c}$

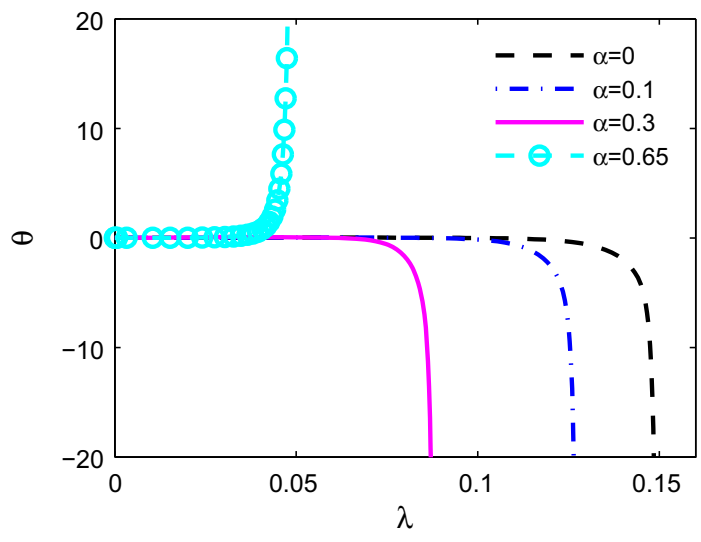

(b) $\theta_{0}>\theta_{c}$

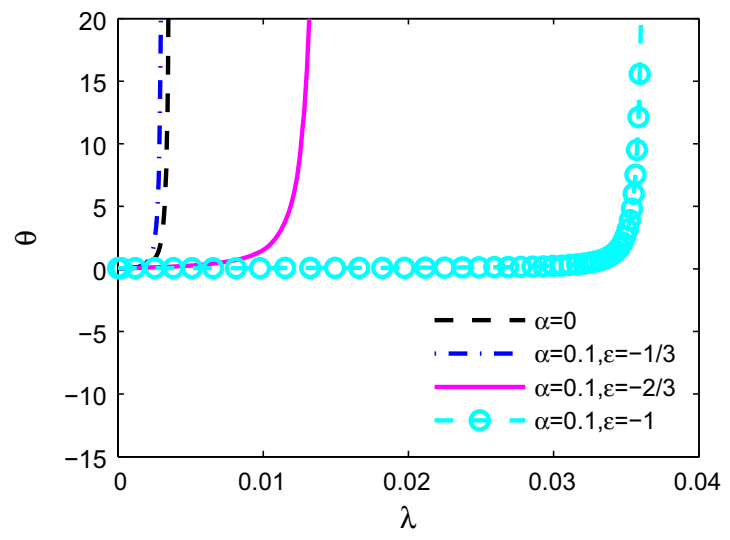

(d) $\varepsilon=-1 / 3, \theta_{0}>\theta_{c}$

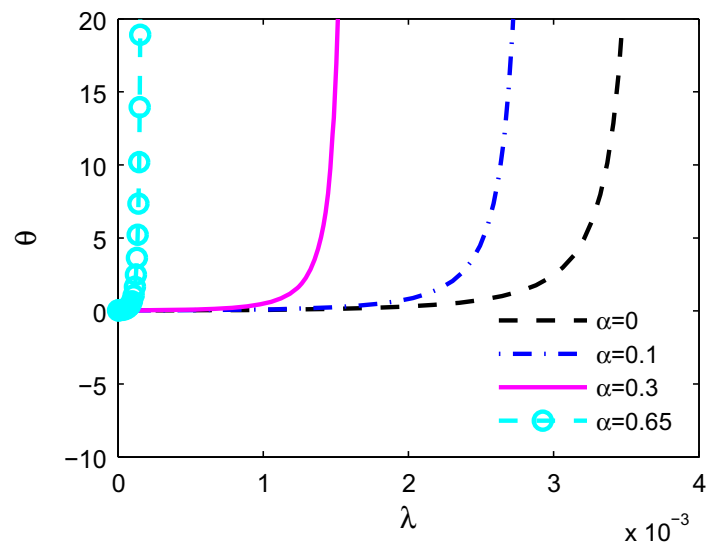

Fig. 7 The evolution of expansion scalar $(\theta)$ with normalisation constant $(\alpha)$ without any initial shear and rotation

rotation. The exact behaviour of geodesic focussing as well as defocussing can easily be visualised in the evolution of ESR variables as presented below. Using the velocity vector field $u_{i}=(\dot{t}, \dot{r}, \dot{\phi})$ in Eqs. (7)-(9), the ESR variables can be represented as functions of $r$. It leads to the following expression for the expansion scalar:

$\theta= \pm \frac{\left[\left(4 r^{2}\left(E^{2}-1\right)+6 M r-2 Q^{2}\right) r^{3 \varepsilon+1}-3 \alpha r^{2}(\varepsilon-1)\right]}{2 r^{3(1-\varepsilon) / 2}\left[\left(\left(E^{2}-1\right) r^{2}+2 M r-Q^{2}\right) r^{3 \varepsilon+1}+\alpha r^{2}\right]^{1 / 2}}$.

The expression given in Eq. (35) for $\theta$ accommodates the evolution of a geodesic congruence for the fixed value of $E$ and $L$ only. It is evident from Eq. (35) that $\theta \rightarrow \pm \infty$ as the denominator vanishes. It is worth noticing that the denominator of RHS of Eq. (35) corresponds to the radial velocity for a test particle with zero-angular momentum and hence it does not include the case of a non-radial motion of test particles as well as the arbitrary initial conditions subjected to the ESR variables. In order to have a complete analysis of the geodesics deformations, one need to solve the Raychaudhuri equations (30-32) arbitrarily.

In the following, the evolution of the ESR variables is presented for the case of a charged BH as well as SBH surrounded with quintessence background, under the different conditions on the parameters involved. The results obtained are compared with those in the RNBH and SBH backgrounds as well as with the corresponding interesting cases having a non-zero cosmological constant.

We study the deformation in equatorial section i.e. $\theta=$ $\pi / 2$. For further numeric computation of the evolution of expansion scalar with the affine parameter, we have considered $E=0.95, L=6.5$ as it represents the energy and angular momentum per unit mass for the test particle in the innermost circular orbit (ISCO) around a SBH with quintessence when $\varepsilon=-2 / 3$. For initially diverging congruences (i.e. $\theta_{0}>0$ ), there exists a critical value of the expansion scalar $\left(\theta_{c}\right)$ below which there is a focussing (i.e. $\theta \rightarrow-\infty)$ when the normalisation constant $(\alpha)$ and the EOS parameter $(\varepsilon)$ have small magnitude while as the value of $\alpha$ becomes more positive or the value of $\varepsilon$ becomes more neg- 

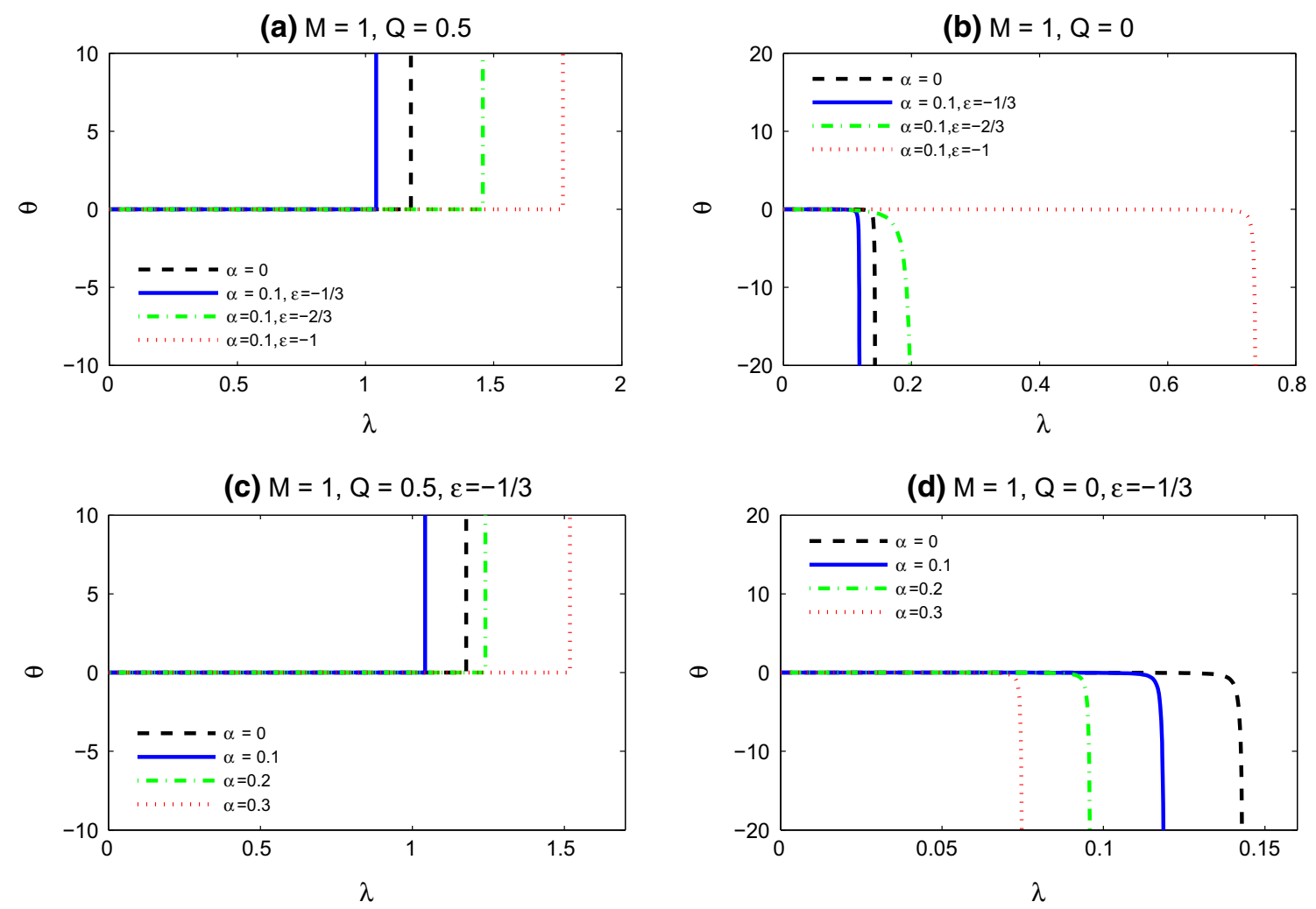

Fig. 8 The evolution of expansion scalar $(\theta)$ with the EOS parameter $(\varepsilon)$ and the normalisation constant $(\alpha)$ without any initial shear and rotation for initially contracting congruences with $\theta_{0}=-0.01$

ative, no focussing is observed as depicted in subsequent figures. It is important to mention that with the change in the initial conditions on shear and rotation, the critical value of the initial expansion scalar will also change. The critical value $\left(\theta_{c}\right)$ of the expansion scalar for SBH is calculated as 0.02902 with $E=0.95, L=6.5$ without initial shear and rotation. For all the figures presented here (Figs. 4, 5, 6 and 7), we have considered $\theta_{0}=0.01$ for $\theta_{0}<\theta_{c}$ and $\theta_{0}=0.1$ for $\theta_{0}>\theta_{c}$. Figure $4 \mathrm{a}-\mathrm{c}$ represent the evolution of expansion scalar for $\theta_{0}<\theta_{c}$ with $\varepsilon=-2 / 3$ for different values of $\alpha$, where (a-c) represent the case of $\mathrm{BH}$, extremal $\mathrm{BH}$ and naked singularity, respectively. Without $\alpha$ geodesics focussing is observed, which converts in defocussing as quintessence appears. The positive increment in the value of $\alpha$ further supports this defocussing, as geodesic defocussing appears earlier with a positive increase in the value of $\alpha$. Figure $4 d-f$ represent the evolution of expansion scalar for initially diverging geodesics with $\theta_{0}>\theta_{c}$ for different values of $\alpha$. In this case, a positive increment in $\alpha$ first delays the defocussing while if one continuously increases the value of $\alpha$ it then supports geodesic defocussing.

Figure 5a, b depict the effect of increasing BH charge $(Q)$ on the evolution of expansion scalar for both cases of initially diverging geodesics. It is observed that there occurs no geodesic focussing due to the presence of quintessence. The defocussing present is further delayed as one compares $\mathrm{BH}$, extremal BH and naked singularity cases, as shown in Fig. 5a. Similar is the effect observed with a negative increment in the value of the EOS parameter $(\varepsilon)$ [47] as shown in Fig. 5c, d. Figure $6 \mathrm{a}, \mathrm{b}$ represent the evolution of the expansion scalar $(\theta)$ for the case $\varepsilon=-1 / 3$, with fixed value of normalisation constant $(\alpha)$. It clearly shows that without initial shear and rotation, the geodesics focus (defocus) as $\theta_{0}<\theta_{c}\left(\theta_{0}>\right.$ $\left.\theta_{c}\right)$. Figures $6 \mathrm{c}-\mathrm{f}$ represent the evolution of expansion scalar $(\theta)$ for $\varepsilon=-2 / 3$ and -1 , respectively, with fixed value of normalisation constant $(\alpha)$. It shows that without initial shear and rotation geodesics defocus even if $\theta_{0}<\theta_{c}$. The presence of an initial shear assists focussing in all the above mentioned cases. As shown in Fig. 6a, where focussing is already present, the presence of an initial shear accelerates focussing. On the other hand, the presence of an initial rotation favours defocussing. As shown in Fig. 6b-f, in the cases where defocussing is already present, the presence of initial rotation assists it.

Figure 7a, b represent the comparative plots for the evolution of the expansion scalar $(\theta)$ with the EOS parameter $(\varepsilon)$ for SBH surrounded by quintessence. An increment in the negative value of $\varepsilon$ plays a different role for the two cases. The cases of SBH and $\varepsilon=-1 / 3$ are quite similar except that focussing and defocussing both appear earlier for later 
case due to the non-zero value of $\alpha$. Figure 7c, $d$ represent the effect of the normalisation parameter $\alpha$ on focussing and defocussing of congruences for $\varepsilon=-1 / 3$. In fact, an increment in the value of $\alpha$ assists both focussing and defocussing. The evolution of the expansion scalar $(\theta)$ is represented for initially contracting congruences with the EOS parameter $(\varepsilon)$ in Fig. 8a, b and with the normalisation parameter $(\alpha)$ in Fig. 8c, din the background of RNBH and SBH, respectively. One can notice that the role of the negative increment in $\varepsilon$ as well as the positive increment in $\alpha$ is similar to that of the respective cases of initially diverging congruences.

\section{Summary and conclusions}

We have investigated the kinematics of timelike geodesic congruences in the background of a charged $\mathrm{BH}$ surrounded with quintessence. The important conclusions are summarised as follows:

- The spacetime representing a charged BH surrounded by quintessence satisfies the WEC but violates the SEC even in the absence of $\mathrm{BH}$ charge.

- The evolution of ESR variables for timelike geodesic congruences is affected qualitatively as well as quantitatively by the normalisation constant and the EOS parameter along with the $\mathrm{BH}$ charge and mass.

- The presence of BH charge supports the defocussing effect of quintessence as for SBH with quintessence the evolution of $\theta$ is similar to the SBH case for small negative values of $\varepsilon$ while no focussing is observed in the presence of both $Q$ and $\alpha$. However, with the increase in negative $\varepsilon$ value, the nature of the evolution shifts towards the corresponding de Sitter spacetimes.

- The normalisation constant behaves like cosmological constant. For initially converging congruences, a positive increment in $\alpha$ always assists geodesic focussing.

- For initially diverging congruences, the positive increment in $\alpha$ assists defocussing when the initial expansion is greater than its critical value for the SBH case. However, for the other case when the initial expansion is smaller than its critical value, a similar increment in $\alpha$ assists geodesic focussing though the congruences defocus as $\alpha$ value is increased further.

The study of the accretion disk formation around the rotating analogue of the $\mathrm{BH}$ spacetimes used in this study would be important astrophysically in view of the permissible range of the quintessence parameters $\varepsilon$ and $\alpha$. Hence by looking into the future observations of ISCOs and accretion onto the BHs, this study might be helpful to constrain the various parameters involved therein from the cosmological view point. In addition to this, the study of null geodesic flows in the background of such BH spacetimes would be useful to provide a more detailed understanding of these spacetimes. We intend to report on these issues in near future.

Acknowledgements The authors are thankful to the anonymous referee for useful suggestions and comments that helped to improve the presentation of the manuscript significantly. The authors are thankful to Prof. M. Sami for useful discussions. One of the authors, HN, would like to thank Department of Science and Technology, New Delhi for financial support through Grant No. SR/FTP/PS-31/2009.

Open Access This article is distributed under the terms of the Creative Commons Attribution 4.0 International License (http://creativecomm ons.org/licenses/by/4.0/), which permits unrestricted use, distribution, and reproduction in any medium, provided you give appropriate credit to the original author(s) and the source, provide a link to the Creative Commons license, and indicate if changes were made. Funded by $\mathrm{SCOAP}^{3}$.

\section{References}

1. J.B. Hartle, An Introduction to Einstein's General Relativity (Pearson Education Inc., Singapore, 2003)

2. S. Chandrashekhar, The Mathematical Theory of Black Holes (Oxford, 1992)

3. E. Poisson, A relativist's toolkit: the mathematics of black hole mechanics (Cambridge University Press, Cambridge, 2004)

4. M. Blau, Lecture Notes on General Relativity (2015). http://www. blau.itp.unibe.ch/Lec-turenotes.html

5. R.M. Wald, General Relativity (University of Chicago Press, Chicago, 1984)

6. B.F. Schutz, A First Course in General Relativity (Cambridge University Press, Cambridge, 1985)

7. A. Einstein, Die feldgleichungen der Gravitation, Sitzung der physikalisch-mathematischenklasse, vol 25 (1915), p 844

8. K. Schwarzschild, Über das Gravitationsfeld eines Massenpunktes nach der Einsteinschen Theorie, Sitzungsber. Preuss. Akad. Wiss. Berlin (Math. Phys. Tech., 1916), p 189

9. H. Reissner, Ann. Phys. (Leipz.) 50, 106 (1916)

10. G. Nordström, Proc. K. Ned. Akad. Wet. 20, 1238 (1918)

11. R.P. Kerr, Phys. Rev. Lett. 11, 237 (1963)

12. E.T. Newman, R. Couch, K. Chinnapared, A. Prakash, R. Torrence, J. Math. Phys. 6, 918 (1965)

13. C.W. Misner, K.S. Thorne, J.A. Wheeler, Gravitation (W. H. Freeman and Company, New York, 1973)

14. E. Hackmann, Geodesic Equations in black hole spacetimes with cosmological constant Ph.D. thesis, Universität Bremen (2010)

15. E. Hackmann, C. Lammerzahl, Phys. Rev. D 78, 024035 (2008)

16. E. Hackmann, C. Lammerzahl, Phys. Rev. Lett. 100, 171101 (2008)

17. M. Jamil, S. Hussain, B. Majeed, Eur. Phys. J. C 75(1), 24 (2015)

18. V. Diemer, J. Kunz, C. LÃd'mmerzahl, S. Reimers, Phys. Rev. D 89(12), 124026 (2014)

19. S. Grunau, B. Khamesra, Phys. Rev. D 87(12), 124019 (2013)

20. S. Fernando, Gen. Rel. Grav. 44, 1857-1879 (2012)

21. J.L. Synge, Ann. Math. 35, 705 (1934)

22. F.A.E. Pirani, Acta Phys. Polon. 15, 389-405 (1956)

23. G.F.R. Ellis, H. van Elst, (1997). arXiv:gr-qc/9709060

24. S. Ghosh, S. Kar, H. Nandan, Phys. Rev. D 82, 024040 (2010)

25. R. Koley, S. Pal, S. Kar, Am. J. Phys. 71, 1037-1042 (2003)

26. R. Uniyal, H. Nandan, K.D. Purohit, Mod. Phys. Lett. A 29, 1450157 (2014) 
27. R. Uniyal, N.C. Devi, H. Nandan, K.D. Purohit, Gen. Rel. Grav. 47, 2-16 (2015)

28. R.S. Kuniyal, R. Uniyal, H. Nandan, A. Zaidi, Astrophys. Space Sci. 357, 92 (2015)

29. R. Uniyal, A. Biswas, H. Nandan, K.D. Purohit, Phys. Rev. D 92(8), 084023 (2015)

30. T. Padmanabhan, Phys. Rep. 380, 235 (2003)

31. J.V. Cunha, J.S. Alcaniz, J.A.S. Lima, Phys. Rev. D 69, 083501 (2004)

32. R.R. Caldwell, Phys. Lett. B 545, 23-29 (2002)

33. L.P. Chimento, R. Lazkoz, Phys. Rev. Lett. 91, 211301 (2003)

34. R.R. Caldwell, R. Dave, P.J. Steinhardt, Phys. Rev. Lett. 80, 15821585 (1998)

35. V. Sahni, L.-M. Wang, Phys. Rev. D 62, 103517 (2000)

36. S. Capozziello, V.F. Cardone, E. Piedipalumbo, C. Rubano, Class. Quant. Grav. 23, 1205-1216 (2006)

37. A. Vikman, Phys. Rev. D 71, 023515(2005)
38. T. Chiba, T. Okabe, M. Yamaguchi, Phys. Rev. D 62, 023511 (2000)

39. R.J. Scherrer, Phys. Rev. Lett. 93, 011301 (2004)

40. H. Wei, R.-G. Cai, D.-F. Zeng, Class. Quant. Grav. 22, 3189-3202 (2005)

41. G.-B. Zhao, J.-Q. Xia, M. Li, B. Feng, X. Zhang, Phys. Rev. D 72, 123515 (2005)

42. L.P. Chimento, M.I. Forte, R. Lazkoz, M.G. Richarte, Phys. Rev. D 79, 043502 (2009)

43. V.V. Kiselev, Class. Quant. Grav. 20, 1187-1198 (2003)

44. S. Fernando, Gen. Rel. Grav. 45, 2053-2073 (2013)

45. A. Dasgupta, H. Nandan, S. Kar, Phys. Rev. D 85, 104037 (2012)

46. A. Dasgupta, H. Nandan, S. Kar, Phys. Rev. D 79, 124004 (2009)

47. H. Nandan, N.M. Bezares-Roder, H. Dehnen, Class. Quant. Grav. 27, 245003 (2010) 